\title{
Orienting behavior and discriminative proficiency of monkeys'
}

\author{
W. A. PIEPER, ${ }^{2}$ F. J. CHIAVERINI, AND V. J. POLIDORA, \\ REGIONAL PRIMATE RESEARCH CENTER, UNIVERSITY OF \\ WISCONSIN, Madison, Wis. 53706
}

Seventeen test-sophisticated rhesus monkeys were trained to discriminate between pairs of visual metric patterns in an automated apparatus (Wisconsin Automated Test Apparatus, WATA) and the Wisconsin General Test Apparatus (WGTA). Regardless of the previous test experience of the animals (WATA or WGTA) their performance in the WATA was consistently superior to that obtained in the WGTA. This finding was interpretated to be the result of design features in the WATA that tended to insure appropriate orienting behavior on each trial.

Previous research from this laboratory (Polidora, 1966) has shown that the proficiency with which experimentally sophisticated monkeys discriminate between two visual metric patterns is almost completely determined by the degree to which the patterns differ. That is, discriminative performance was directly related to the extent to which the two patterns, when translated, had unique areas. Multiple regression and discriminant function analyses of the data revealed furthermore that this "Unique Area" dimension accounted for almost all the nonresidual variance, to the exclusion of dimensions based on disparity of the two patterns with respect to more classical expressions of pattern complexity (e.g., number of sides or angles) or geometrical configurations (e.g., moments of area).

Because these earlier experiments were performed in an automated apparatus, however, the utility of these results to other investigators was indeterminate due to the uniqueness of the apparatus. The experiments reported here were designed to determine the extent to which a Unique Area dimension would also predict performance of monkeys discriminating the same visual metric patterns in the traditional apparatus for monkeys, the Wisconsin General Test Apparatus (WGTA).

\section{EXPERIMENT 1}

Method. The Ss were the same 13 (eight male) rhesus monkeys (Macaca mulatta) which had been used in the earlier series of experiments (see Polidora, 1966, for references) with the automated apparatus (Wisconsin Automated Test Apparatus, WATA). Therefore, not only were the Ss extremely test-sophisticated, having completed thousands of two-choice, visual metric pattern discrimination problems, but they also had recently solved in the WATA the same 768 problems which they were to receive in the present experiment in a WGTA.

The apparatus was a standard WGTA with a modified formboard. Two wedge-shaped holders were positioned such that $1.5 \times 1.5$ in. photographs mounted in transparent plastic carriers covered the two reward wells. When $S$ pushed the correct stimulus pattern, the reward (raisin or corn) was exposed in the well.

The stimuli were photographs of the same visual metric patterns used in the previous experiment. A pattern was formed by lighting several of the 16 $3 / 8$ in. square lights mounted in a $4 \times 4$ array (see Polidora, 1966, for a more complete description). Photographs of the stimuli were true-to-scale, and since S's viewing distances in the WGTA and in the WATA were similar, retinal size of stimuli were also. Pairs of patterns constituted a discrimination problem, and each problem was presented for seven noncorrection trials, seven problems per daily session. After 532 problems the photographic facsimilies were replaced with two 16-element light displays identical to those used in the WATA. Push responses to the display were again required to expose the reward, and all other procedural details were the same. After an adaptation period to the lighted displays, Ss received 236 problems.

Results. In the preceding experiment in the WATA mean percentage correct responses on trials $2-7$ by all Ss on the total 768 problems was $71.3 \%$. When presented with the same problems in the WGTA, performance dropped to near chance $(52.6 \%)$, a statistically reliable difference $(F=41.14, \mathrm{df}=1 / 12, \mathrm{p}<.001)$. When lighted displays were used, performance did not markedly change.

\section{EXPERIMENT 2}

In Experiment 1 all Ss had first been extensively trained in the WATA and then adapted and tested in the WGTA. Because this sequence of treatments might have artifactually depressed performance in the WGTA due to percentage correct responses on Trials $2-7$ by all $S s$ on the total 768 problems imposed in Experiment 2. In addition, Ss were tested in both apparatuses on the same day on the same problems to determine if a discrimination acquired in the WATA could be performed in the WGTA.

Method. Five adult male rhesus monkeys (11 to 19 years of age) were used. Each had participated in numerous experiments in the WGTA, and each was highly proficient at performing simultaneous visual discrimination learning-set problems, although Ss had had no previous experience with visual metric pattern stimuli.

After being adapted to the WATA, each S received 116, 25-trial problems drawn randomly from those used in Experiment 1. Next, Ss received in the WGTA (lighted displays) 235, 10-trial problems followed by 100, 25-trial problems. Finally, a transfer test was run on two consecutive days. Four discrimination problems were selected, each consisting of a pair of patterns which were highly discriminable (i.e., many unique elements). On the first day the Ss received two, 25-trial problems in the WATA, and were then taken immediately to the WGTA to perform the same two problems for 25 additional trials. On the second test day the Ss received two, 25-trial problems in the WGTA and then were taken to the WATA for the transfer test.

Results. Despite the fact that these WGTA-sophisticated Ss received 4,850 trials ( 335 problems) in the WGTA and only 2,900 trials (116 problems) in the WATA, the pattern of the results was similar to that obtained in Experiment 1 with WATA-sophisticated Ss. Overall percentage correct responses was $65.0 \%$ in the WATA and only $51.8 \%$ in the WGTA.

In the final portion of Experiment 2, when Ss received in one session the same problems in both apparatuses, the same pattern of results was once again obtained. When original learning was in the WGTA, followed by the same problems in the WATA, performance (percentage correct responses on trials 2-25) was 53.3 and 65.0 ; for the converse sequence, $60.4 \%$ correct during acquisition in WATA was followed by $56.3 \%$ on the same problems in the WGTA $(\mathrm{F}=5.28, \mathrm{df}=1 / 28, \mathrm{p}<.05)$.

It should be noted also that in both Experiments 1 and 2 near chance performance was obtained on all problems in the WGTA regardless of the number of unique elements. In the WATA, although mean performance was near $65 \%$, the range for individual problems was between near chance and over $80 \%$, the number of unique elements in a problem being positively related to performance.

Discussion. These results can be summarized with one succinct statement: Regardless of the test experience of $\mathrm{S}$, visual metric pattern discrimination problems could not be solved by monkeys in the WGTA, but in the WATA, performance consistently exceeded chance and was a direct function of the number of unique elements in the pair of patterns constituting a problem.

Our interpretation of this result relates to two features of the WATA, features not found in the WGTA, which tend to insure appropriate and necessary orienting behavior on each trial. In the WATA, $S$ is required to initiate each trial by inserting his head into a formfitting face mask, thereby interrupting a photobeam. Only when the photobeam is interrupted are the displays lighted so that $\mathrm{S}$ may view them through the eyeholes in the face mask. Since these two features (trials initiated by $S$ and visual orientation restricted to the stimuli by the eyeholes of the mask) are unique to the WATA, and since all other potentially important details of apparatus design and procedure (e.g., spatial contiguity of sites of stimuli and responses) would seem to be common to both apparatuses, we conclude that one or both of these features are responsible for the effects obtained. Apparently monkeys are capable of learning to make adequate orienting responses in the WGTA when they are required to discriminate relatively distinctive stimuli such as stereometric objects or simple patterns. When monkeys are required to discriminate on the basis of more subtle or obscure differences between visual patterns. such as the differences between visual metric patterns. the present data indicate that 
even WGTA-sophisticated Ss are incapable of mustering sufficiently consistent orienting behavior in the WGTA to insure proficient discrimination performance.

\section{REFERENCES}

POLIDORA, V. J. Stimulus correlates of visual pattern d:scrimination by monkeys: Multidimensional analyses. Percept. \& Psychophys., 1966, 1, $405-414$.
NOTE

1. This investigation was supported in part by a Public Health Service fellowship 5-F3-MH-17,387-02 (PS), to the senior author, NIH Grant FR-0167 and research contract DAAA $15-67-0296$ from the U.S. Army Chemical R \& D Laboratories. In conducting the research reported herein, the investigators adhered to the "Principles of Laboratory Animal Care" as established by the National Society for Medical Research.

2. Now at the Yerkes Regional Primate Research Center, Emory University, Atlanta, Georgia 30322. 